Aim of the study was to evaluate the potential role of regulatory and proinflammatory cytokines IL-23 and IL-17 as Th17 lymphocyte activity markers in relation to invasiveness in laryngeal cancer.

Material and methods: The immunological analysis was conducted in 50 patients treated for squamous cell laryngeal carcinoma and 30 healthy volunteers as controls. The levels of IL-23 and IL-17 in supernatants of purified peripheral blood mononuclear cell cultures were determined by using the enzyme-linked immunosorbent assay (ELISA). The clinicomorphological criteria included pTNM, stage, $G$, and the total tumour front grading (TFG) score.

Results: Our data demonstrated higher concentrations of IL-23 in patients as compared to controls ( $p=0.0001$ ). No statistical difference for IL-17 in these groups was observed. Our study revealed significant dependences in IL-23 expression on PT $(p=0.04)$, histological differentiation $(p=0.04)$, and TFG total score $(p=0.02)$. Advanced tumours (pT3-pT4) with higher grade (G2-G3) and higher invasiveness (> 14 TFG points) were characterised by elevated IL-23 levels in PBMC supernatants. Our data did not indicate a relationship between cytokine levels and three- and five-year survival. However a tendency towards lower content of IL-23 in PBMC cultures in patients who lived longer than five years after treatment was noted. The relationships between IL-17 level in PBMC cultures and clinicomorphological and prognostic parameters have not been disclosed.

Conclusions: The results of this study suggest the importance of regulatory cytokine IL-23 in determining the aggressive potential of laryngeal carcinomas.

Key words: laryngeal carcinoma, IL-23, IL-17, tumour front grading.

Contemp Oncol (Pozn) 2015; 19 (3): 195-200 DOI: $10.5114 /$ wo.2015.51612

\section{Expression of Th17 cell population regulatory cytokines in laryngeal carcinoma - Preliminary study}

\author{
Renata Kopta ${ }^{1}$, Marcin Mochocki ${ }^{1}$, Piotr Morawski ${ }^{1}$, \\ Ewa Brzezińska-Błaszczyk², Iwona Lewy-Trenda ${ }^{3}$, Student Scientific Circle ${ }^{4 *}$, \\ Katarzyna Starska ${ }^{5}$
}

*Student Scientific Circle of Laryngological Immunobiology: Khaliunaa Lkhagva, Katarzyna Kolary, Agnieszka Pomykała, Joanna Nestorowicz, Jakub Miazga, Mateusz Tomaszewski

${ }^{1}$ Department of Otolaryngology, Żeromski Specialist Hospital, Kraków, Poland ${ }^{2}$ Department of Experimental Immunology, Medical University of Lodz, Lodz, Poland ${ }^{3}$ Department of Pathology, Medical University of Lodz, Lodz, Poland

${ }^{4}$ Student Scientific Circle of Laryngological Immunobiology, I Department of Otolaryngology and Laryngological Oncology, Medical University of Lodz, Lodz, Poland

${ }^{5}$ Department of Otolaryngology and Laryngological Oncology, Medical University of Lodz, Lodz, Poland

\section{Introduction}

Th17, a population of T helper lymphocytes, producing interleukin (IL)-17, a proinflammatory cytokine that enhances T-cell priming and stimulates the production of other interleukins and molecules such as IL-1 $\beta$, IL-6, TNF, NOS2 , as well as chemokines. Th17 cells are in turn produced as a result of naïve CD4+ T-cell stimulation by IL-23, IL-6, and TGF- $\beta 1$. The most notable role of $\mathrm{IL}-17$ is its involvement in inducing and mediating proinflammatory responses, e.g. production of many cytokines, chemokines, and prostaglandins, enhancement of dendritic cell maturation, and stimulation of macrophages [1]. Interleukin 23 is a heterodimeric cytokine produced by dendritic cells and macrophages. The IL-23 receptor complex is expressed in a number of cells, including natural killer cells, monocytes, macrophages, dendritic cells, and CD4+ T cells [1]. It appears that the same processes and relationships that are involved in the inflammation may also stimulate cancer growth [2]. Nevertheless, the participation of Th17 cells in anti-tumour immunity remains poorly understood. Cytokines IL-17 and IL-23 have pleiotropic effects. They may be tumour-promoting or tumour-suppressing factors [3-5]. For instance, IL-17 promotes an anti-tumour cytotoxic T-cell response leading to tumour regression. On the other hand, by facilitating angiogenesis and egress of tumour cells from the primary focus, IL-17 promotes tumour growth [6, 7]. It is believed that tumour growth, particularly in the early stages, is triggered by the IL-17 inflammatory effect on the activity of IL- 8 and IL-6, and is enhanced by the infiltration of immunocompetent cells to the tumour. While IL-17 can promote the cells leading to tumour regression, IL-23 contributes to the impairment of specific neoplastic cytotoxic CD8+ T lymphocytes and reduces cell infiltration into the transformed tissue [2, 6]. IL-23 may promote tumour progress by activation of neutrophils and macrophages, as well as enhancement of angiogenesis. Moreover, by promoting upregulation of the matrix metalloprotease MMP9, IL-23 acts as a tumour stimulating factor [2]. Numerous data indicate the suppressive effect of cytokines secreted by Th17 cells [8-10]. Assessing the interaction between cancer cells and immune cells, including Th17 cells and secreted IL-17 and IL-23 in relation to tumour aggressiveness, is a leading issue in oncological immunology. Nevertheless, little is known about the possible function of Th17 lymphocytes in the development and progression of laryngeal carcinoma. 
The aim of this study was to evaluate the potential role of IL-23 and IL-17, the regulatory cytokines of Th17 cell populations, and to assess the relationships with tumour invasiveness according to clinicomorphological features such as PTNM, G, stage, and tumour front grading score in squamous cell carcinoma.

\section{Material and methods}

The study involved a group of 50 patients (48 men, 2 women) with laryngeal squamous cell carcinoma (aged 45-79 years; mean age $62.5 \pm 8.3$ years) and a control group of 30 healthy volunteers (aged 40-65 years; mean age $54.2 \pm 5.9$ years). The criteria for patient participation in this study were as follows: 1) pathologically confirmed diagnosis of carcinoma planoepitheliale; 2) primary surgical resection without receiving prior immuno-, radio-, or chemotherapy; and 3) absence of distant metastasis.

The clinicomorphological characteristics of the studied group are shown in Table 1.

\section{Histological classification and morphological features}

Morphological assessment of tumours was performed on archival paraffin-embedded tissue samples. All specimens were estimated according to the criteria conducted in accordance with the AJCC TNM 2010 classification for laryngeal cancer [11]. Morphological analysis was performed on $\mathrm{H} \& \mathrm{E}$-stained sections from the most invasive, peripheral zones of the cancer infiltration, according to tumour front grading (TFG), a reliable and precise histological method for the evaluation of neoplastic progress [12]. The histological parameters: three tumour-related features (cytoplasmic differentiation, nuclear polymorphism, number of mitoses) and three adjacent stroma-related character-

Table 1. Clinicopathological characteristics of laryngeal cancers

\begin{tabular}{lcc}
\hline Surgical & Variable & Cases, $\boldsymbol{n}(\%)$ \\
treatment & Total laryngectomy & $33(66)$ \\
& Partial laryngectomy & $17(34)$ \\
& Neck dissection (-) & $25(50)$ \\
& Neck dissection (+) & $25(50)$ \\
& Selective neck dissection & $21(42)$ \\
& Radical neck dissection & $4(8)$ \\
Tumour size & pT1 & $6(12)$ \\
(pT status) & pT2 & $13(26)$ \\
& pT3 & $16(32)$ \\
Nodal & pT4 & $15(30)$ \\
metastases & pNo & $40(80)$ \\
(pN status) & pN1-3 & $10(20)$ \\
Degree of & & \\
differentiation & G1 & $9(18)$ \\
(grade) & G2 & $34(68)$ \\
TFG total score & G3 & $7(14)$ \\
& $6-13$ points & $19(38)$ \\
& $14-17$ points & $16(32)$ \\
Survival & $18-21$ points & $15(14)$ \\
& $<3$ years & $8(16)$ \\
& $3-5$ years & $11(20)$ \\
& $\geq 5$ years & $31(64)$
\end{tabular}

istics of the peripheral edge of tumour infiltration (mode of invasion, depth of invasion, and plasmalymphocytic infiltration) were assessed in at least five different regions of the peripheral part of the tumour (magnification 200x, number of mitoses magnification 400x). Each factor was graded according to a scale ranging from one to four. The numeric morphological TFG score was computed as a sum of six morphological features, with a maximum score of 24 points. According to the TFG total score and results for laryngeal carcinomas, tumours in this study were divided into three groups: 6-13, 14-17, 18-21 TFG points.

\section{Lymphocyte isolation and ELISA tests for IL-23 and IL-17 measurement}

For peripheral blood mononuclear cell (PBMC) isolation the venous blood of each patient was obtained $(10 \mathrm{ml})$ and transferred to test tubes containing heparin $(10 \mathrm{U} / \mathrm{ml})$. The control blood samples were obtained from 30 healthy volunteers without a history of malignancies or autoimmune disorders. PBMCs were isolated by Ficoll-Hypaque density gradient and resuspended in RPMI 1649 medium to obtain the concentration of $1 \times 10^{6}$ cells $/ \mathrm{ml}$. The recovered cells were checked and counted for viability with Trypan Blue staining method. The isolated PBMC cultures were incubated for 21 hours at $37^{\circ} \mathrm{C}$ in a humidified atmosphere with $5 \% \mathrm{CO}_{2}$ (Cellstar Incubator) in 96-well plates in a final volume of $0.2 \mathrm{ml}$ (per well). The supernatants of cultures were collected and the secretion pattern of IL-23 and IL-17 was measured with specific enzyme-linked immunosorbent assay ELISA Kit (R\&D Systems, Inc.; Minneapolis, MN, USA) according to the manufacturer's instructions. Absorbance was measured with an ELISA reader (Multiscan RC 351). The sensitivity of this assay was $6.8 \mathrm{pg} / \mathrm{ml}$ for IL-23 and $<15 \mathrm{pg} / \mathrm{ml}$ for IL-17. The investigations were performed with the approval of the Bioethical Commission of the Medical University of Lodz and the National Science Council, Poland (No. RNN/60/13/KE).

\section{Statistical analysis}

The statistical analyses were performed using the IBM SPSS STATISTICS 21 (Business Machines Corp., USA) software package. Distributions of quantitative variables were described using means and standard deviations. Since levels of IL-17 and IL-23 expression did not show normal distribution (according to the results of Shapiro-Wilk normality test) the non-parametric statistical tests: Mann-Whitney $U$ test and Kruskal-Wallis test with post hoc multiple comparisons with Bonferroni correction, were used to identify the relationship between cytokines expression and clinicopathological parameters. A p-value of less than 0.05 was considered as statistically significant.

\section{Results}

Analysis of IL-23 and IL-17 secretion in cases and control group

Analysis of the data showed a significant difference of the average concentration of IL-23 in the supernatant of PBMCs obtained from patients with squamous cell laryn- 
geal carcinoma, as compared to the level of this cytokine in the control group $(p<0.0001)$. The mean concentration of IL-23 in cases was higher in comparison with cytokine secretion in the control group $(198.3 \pm 303.9 \mathrm{pg} / \mathrm{ml}$ vs. 12.6 $\pm 3.7 \mathrm{pg} / \mathrm{ml}$, for cases and controls, respectively). No significant differences were disclosed between IL-17 mean concentrations in both groups, although slightly higher values of IL-17 concentration were noted in the control group (9.9 $\pm 24.0 \mathrm{pg} / \mathrm{ml}$ vs. $12.9 \pm 43.0 \mathrm{pg} / \mathrm{ml}$, for patients and controls, respectively).

\section{The association of IL-23 and IL-17 expression with clinicomorphological features}

Subsequently, the pattern of IL-23 and IL-17 expression in purified PBMC cultures from patients treated with laryngeal carcinoma with clinicomorphological parameters was compiled. The present study demonstrated that the increased expression of IL-23 in laryngeal carcinoma cases was an indicator of more advanced neoplastic lesions. Statistical analysis confirmed significant relationship between IL-23 level and local extension of the tumour pT $(p=0.04)$. The presence of the higher content of IL-23 in supernatants of PBMCs was more frequent for tumours with higher $\mathrm{pT}$ feature. Moreover, a significant difference between $\mathrm{pT} 2$ and $\mathrm{pT} 3$ subgroups was disclosed $(p=0.03)$. Similarly, statistical analysis identified a positive association between IL-23 level and histological differentiation $(p=0.04)$ and between the G1 and G3 subgroups $(p=0.02)$. The higher concentration of IL-23 in PBMC cultures was more characteristic for less differentiated tumours. No statistical dependences were noted for $\mathrm{pN}$ and stage. However, a clear tendency towards higher values for IL-23 in laryngeal carcinomas characterised by the nodal metastases (pN1-3) and higher clinical advancement (SIII-IV) was noted. Statistical evaluation of the quantitative analysis results and the clinicomorphological features of laryngeal carcinomas showed that the expression of
IL-23 in PBMCs isolated from patients was significantly different depending on the total score of tumour front grading classification $(p=0.02)$. The presence of the higher content of IL-23 in supernatants of PBMCs was more frequent for tumours with more aggressive behaviour determined by higher total score of TFG scale (characterised by $14-21$ points) in comparison with less invasive carcinomas (not exceeding 6-13 points in TFG) $(p=0.02)$. Unfortunately, no correlation between the level of IL-17 and the aggressiveness of tumours studied according to both, pTNM classification and TFG scale was confirmed. The IL-23 and IL-17 expression in PBMC cultures in the laryngeal carcinoma group studied and the statistical analysis results are shown in Table 2 and Table 3.

\section{The association of IL-23 and IL-17 expression and} three-year and five-year survival

The relationship of IL-23 and IL-17 level in supernatants of purified PBMCs with three-year and five-year survival of patients with laryngeal carcinoma was analysed. Statistical analysis did not disclose significant differentiation of both, IL-23 and IL-17 concentration in relation to patient survival. However, a tendency towards lower content of IL23 in PBMC cultures in patients who lived longer than five years after treatment as well as higher values for IL-17, for whom the survival period was over three years, was noted. The IL-23 and IL-17 levels in PBMC cultures in patients with laryngeal carcinoma and statistical analysis results are shown in Figs. 1 and 2.

\section{Discussion}

In recent decades, numerous studies have confirmed the dependence of immune system activity and both, the development and aggressiveness of cancers of various origins. Nevertheless, little is known about the possible role of IL-17 and IL-23 in the development and progression of laryngeal carcinomas.

Table 2. Associations between clinicopathological characteristics and expression of IL-23

\begin{tabular}{|c|c|c|c|c|}
\hline & Variable & IL-23 [pg/ml] & $p^{\mathrm{a}}$ & $p^{b}$ \\
\hline Tumour size (pT status) & $\begin{array}{l}\text { pT1 } \\
\text { pT2 } \\
\text { pT3 } \\
\text { pT4 }\end{array}$ & $\begin{array}{c}174.5 \pm 224.6 \\
111.9 \pm 175.7 \\
288.2 \pm 404.2 \\
230.1 \pm 241.8\end{array}$ & 0.04 & $\begin{array}{c}\text { pT2 vs. pT3 } \\
p=0.03\end{array}$ \\
\hline Nodal metastases (pN status) & $\begin{array}{l}\text { pNO } \\
\text { pN1-3 }\end{array}$ & $\begin{array}{l}190.1 \pm 288.2 \\
235.0 \pm 336.9\end{array}$ & $>0.05$ & $>0.05$ \\
\hline Degree of differentiation (Grade) & $\begin{array}{l}\text { G1 } \\
\text { G2 } \\
\text { G3 }\end{array}$ & $\begin{array}{c}86.8 \pm 162.6 \\
221.7 \pm 355.6 \\
211.9 \pm 272.5\end{array}$ & 0.04 & $\begin{array}{l}\text { G1 vs. G3 } \\
p=0.02\end{array}$ \\
\hline TFG total score & $\begin{array}{l}6-13 \text { points } \\
14-17 \text { points } \\
18-21 \text { points }\end{array}$ & $\begin{array}{l}116.2 \pm 198.3 \\
253.4 \pm 379.4 \\
230.1 \pm 303.9\end{array}$ & 0.02 & $\begin{array}{c}6-13 \text { vs. } 14-17 \\
p=0.02\end{array}$ \\
\hline Survival & $\begin{array}{l}<3 \text { years } \\
3-5 \text { years } \\
\geq 5 \text { years }\end{array}$ & $\begin{array}{c}238.9 \pm 336.9 \\
221.7 \pm 349.1 \\
155.4 \pm 242.6\end{array}$ & $>0.05$ & $>0.05$ \\
\hline
\end{tabular}

Results are given as mean \pm standard deviation

${ }^{a}$ Differences between two groups were evaluated with Mann-Whitney $U$ test, differences between three or four groups were evaluated with Kruskal-Wallis ${ }^{b}$ Multiple comparison for Kruskal-Wallis test with Bonferroni correction 
Table 3. Associations between clinicopathological characteristics and expression of IL-17

\begin{tabular}{|c|c|c|c|c|}
\hline & Variable & IL-17 [pg/ml] & $p^{a}$ & $p^{b}$ \\
\hline Tumour size (pT status) & $\begin{array}{l}\text { pT1 } \\
\text { pT2 } \\
\text { pT3 } \\
\text { pT4 }\end{array}$ & $\begin{array}{c}5.5 \pm 24.0 \\
12.3 \pm 32.1 \\
6.7 \pm 23.7 \\
10.9 \pm 26.8\end{array}$ & $>0.05$ & $>0.05$ \\
\hline Nodal metastases (pN status) & $\begin{array}{c}\text { pNO } \\
\text { pN1-3 }\end{array}$ & $\begin{array}{c}10.7 \pm 31.3 \\
8.8 \pm 23.8\end{array}$ & $>0.05$ & $>0.05$ \\
\hline Degree of differentiation (grade) & $\begin{array}{l}\text { G1 } \\
\text { G2 } \\
\text { G3 }\end{array}$ & $\begin{array}{c}6.6 \pm 23.7 \\
10.6 \pm 28.4 \\
10.9 \pm 23.8\end{array}$ & $>0.05$ & $>0.05$ \\
\hline TFG total score & $\begin{array}{l}6-13 \text { points } \\
14-17 \text { points } \\
18-21 \text { points }\end{array}$ & $\begin{array}{c}10.4 \pm 24.0 \\
10.7 \pm 30.2 \\
4.0 \pm 21.7\end{array}$ & $>0.05$ & $>0.05$ \\
\hline Survival & $\begin{array}{l}<3 \text { years } \\
3-5 \text { years } \\
>5 \text { years }\end{array}$ & $\begin{array}{c}3.2 \pm 33.0 \\
9.9 \pm 20.1 \\
10.9 \pm 31.3\end{array}$ & $>0.05$ & $>0.05$ \\
\hline
\end{tabular}

Results are given as mean \pm standard deviation

${ }^{a}$ Differences between two groups were evaluated with Mann-Whitney U test, differences between three or four groups were evaluated with Kruskal-Wallis ${ }^{b}$ Multiple comparison for Kruskal-Wallis test with Bonferroni correction

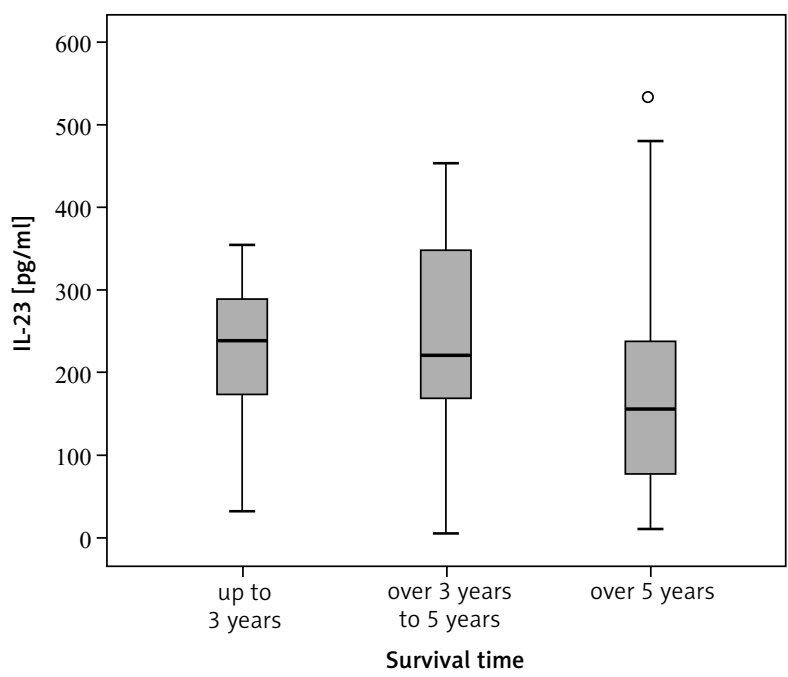

Fig. 1. The concentration of IL-23 in the group studied depending on the 3-year and 5-year survival time

The findings of our study into IL-23 correlate with the results obtained by other authors who studied various types of tumours, and tumours of other localisation [13-16]. It should be stressed that, despite numerous scientific publications on the role of IL-17 and IL-23 in cancer, only a few published works have dealt with tumours of the head and neck [17-19]. Meng et al. [17] assessed the level of IL-17 in tumour-associated macrophages in three groups of patients: with laryngeal squamous cell carcinoma, patients with cancer of adjacent tissues as the control group, and with chronic hypertrophic laryngitis. The expression of the serum level of IL-17 was similar in the three studied groups, while relatively high levels of IL-17 were noted in macrophages in two other assessment methods in patients with LSCC [17]. Kesselring et al. [18] found that lymphocytes Th17 and produced IL-17 have a substantial impact on the carcinogenesis of head and neck squamous

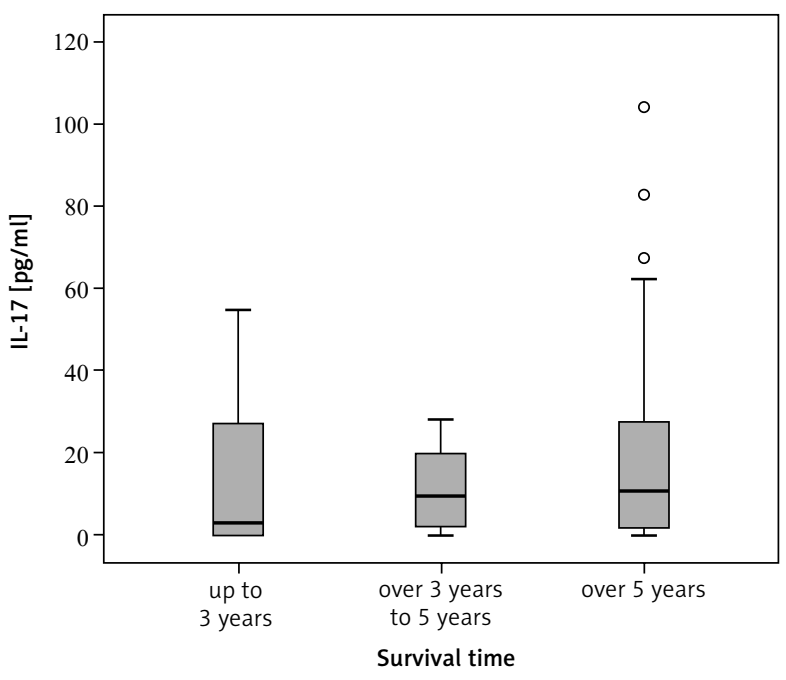

Fig. 2. The concentration of IL-17 in the group studied depending on the 3-year and 5-year survival time

cell carcinoma as well as on their metastasis formation. The tissues of tumour and lymph nodes are infiltrated by a huge number of Th17 cells representing an important fraction of tumour-infiltrating lymphocytes (TILS). According to the above authors, proliferation and angiogenesis of HNSCC are impaired in the presence of Th17 cells. Garley et al. [19] assessed the levels of IL-17E and IL-17A in patients with oral epithelial squamous cell carcinoma. They showed that the levels of both cytokines were higher both in cell supernatants and in the blood serum of the patients compared to a control group of healthy subjects. However, no correlation was found between the protein expression in the cells or the concentration of supernatants of the patients with different stages of disease.

Many authors confirm that an increase in immunocompetent cell activity may be an indirect index of advancement of the neoplastic process [2, 4, 5, 13, 18]. For instance, 
Matowiecka-Karna et al. [13] observed increased levels of IL-6 and IL-23 in the blood serum of patients with gastric cancer as compared to healthy subjects. However, lower levels of both cytokines were found in patients with metastatic gastric cancer. Yaghoobi et al. [14] noted a substantial rise of IL-23 levels in the anal mucosa of HIV-positive patients with anal cancer as compared to patients without anal cancer. Wu et al. [20] observed in patients suffering from early recurrence of primary hepatocellular carcinoma (HCC) higher pre-therapy serum levels of IL-17 than in patients who did not have recurrence after a radical hepatectomy. Zhang et al. [23] also found that the main immunological risk factors for the prognosis of non-small cell lung cancer (NSCLC) are negative CD4 expression and positive IL-17 and Foxp3 expressions. However, opposing results were obtained by Hus et al. [24]. They showed that higher levels of Th17 and IL-17A were associated with less advanced clinical stage of chronic lymphocyte leukaemia (CLL). Th17 and IL-17A levels were lower in patients with adverse prognostic factors. A number of authors underline the possibility of utilising the research for cancer prevention. Yao et al. [25] revealed two genetic variants for IL-23R, rs6682925, and rs10889677, which play a particular role in the pathogenesis of multiple cancers. This was confirmed by Zhou et al. [26], who in their studies pointed to the genetic variant rs10889677, which increases the probability of malignant transformation of a great number of solid tumours.

Multidirectional studies presently being conducted are searching for the mechanisms that induce the onset of carcinogenesis and tumour growth. Some authors indicate the role of equilibrium between IL-12 and IL-23 in maintaining cancer cells in a state of immunological quiescence [3, 27-30]. Kortylewski et al. [3] showed that IL-12 significantly boosts precancerous immunity through stimulation of lymphocytes Th1, differentiation of cells, and cytotoxic stimulation of lymphocytes $T$ and natural killer cell cytotoxicity. Interleukin 23 had pro-cancerous properties, and the expression of IL-23 was induced by Stat3, which is produced mainly by tumour-associated macrophages. Choi et al. [27] in their studies into oncolytic adenoviruses, which inhibit IL-23 expression, pointed out their role in immunotherapy since they may increase antitumour immunity. Cui et al. [29] investigated the level of IL-17A in the microenvironment of colorectal cancer. The IL-17 level gradually increased along with the severity of dysplasia. The level of IL-17 was markedly higher in cases of colorectal cancer. Omrane et al. [30] noted a significant association between IL-17F and IL-23R polymorphism and the location, histology, and architecture of tumours in patients with colorectal cancer (CRC). They also found a significant relationship between the increased level of IL-17F and an increased risk of TNM advanced stage. A number of authors indicate the necessity of further studies into IL-23 since it seems to be a novel appropriate remedy that could be employed in cancer treatment, and the possibility of monitoring IL-23 levels as a novel therapeutic target, which gives hope for the inhibition of cancer progression [16, 31-33].

The results of this study should be interpreted with caution, and they are difficult to compare with the results of other authors due to the different localisation and types of cancers, the use of different study methods (ELISA, Western blot, RT-PCR), and the variety of materials (serum, tissues, cell lines, and laboratory animals). Moreover, our study material included a relatively small group of patients with laryngeal carcinoma. Additional research to confirm these findings in a larger group and further studies with regard to relations with other immunological parameters that affect the function of immunocompetent cells, including Th17 lymphocytes, are mandatory [34]. Regardless of these limitations, the results assessing concentrations of cytokines IL-17 and IL-23 in laryngeal squamous cell carcinoma should be taken into consideration in the discussion on the role of cytokine activity in the advancement and progression of cancer, its prognosis, and selection of an appropriate therapeutic intervention.

The authors declare no conflict of interest.

This work was supported by grants from the National Science Council, Poland (N403 043 32/2326).

\section{References}

1. Gołąb J, Jakóbisiak M. Immunologia. Wydawnictwo Naukowe PWN, Warszawa 2010.

2. Langowski JL, Zhang X, Wu Let, et al. IL-23 promotes tumour incidence and growth. Nature 2006; 442: 461-5.

3. Kortylewski M, Xin H, Kujawski M, et al. Regulation of the IL-23 and IL-12 balance by Stat3 signaling in the tumor microenvironment. Cancer Cell 2009; 15: 114-23.

4. Teng MW, Andrews DM, McLaughlin N, et al. IL-23 suppresses innate immune response independently of IL-17A during carcinogenesis and metastasis. Proc Natl Acad Sci U S A 2010; 107: 8328-33.

5. Nuñez S, Saez JJ, Fernandez D, et al. T helper type 17 cells contribute to anti-tumor immunity and promote the recruitment of $\mathrm{T}$ helper type 1 cells to the tumor. Immunology 2013; 139: 61-7.

6. Murugaiyan G, Saha B. Protumor vs antitumor functions of IL-17. J Immunol 2009; 183: 4169-75.

7. Numasaki M, Fukushi J, Ono M, et al. Interleukin-17 promotes angiogenesis and tumor growth. Blood 2003; 101: 2620-7.

8. Kryczek I, Banerjee M, Cheng P, et al. Phenotype, distribution, generation and functional and clinical relevance of Th17 cells in the human tumor environments. Blood 2009; 114: 1141-9.

9. Kryczek I, Wei S, Szeliga W, Vatan L, Zou W. Endogenous IL-17 contributes to reduced tumor growth and metastasis. Blood 2009; 114: 357-9.

10. Kryczek I, Wu K, Zhao E, et al. IL-17+ regulatory T cells in the microenvironments of chronic inflammation and cancer. J Immunol 2011; 186: 4388-95.

11. Edge SB, Compton CC. The American Joint Committee on Cancer: the 7th edition of the AJCC cancer staging manual and the future of TNM. Ann Surg Oncol 2010; 1471-4.

12. Starska K, Kulig A, Lukomski M. Tumor front grading in prediction of survival and lymph node metastases in patients with laryngeal carcinoma. Adv Med Sci 2006; 51: 200-4.

13. Matowicka-Karna J, Kamocki Z, Polińska B, Osada J, Kemona H. Platelets and inflammatory markers in patients with gastric cancer. Clin Dev Immunol 2013; 2013: 401623.

14. Yaghoobi M, Le Gouvello S, Aloulou N, Duprez-Dutreuil C, Walker F, Sobhani I. FoxP3 overexpression and CD1a+ and CD3+ depletion in anal tissue as possible mechanisms for increased risk of human papillomavirus-related anal carcinoma in HIV infection. Colorectal Dis 2011; 13: 768-73.

15. Chan IH, Jain R, Tessmer MS, et al. Interleukin-23 is sufficient to induce rapid de novo gut tumorigenesis, independent of carcino- 
gens, through activation of innate lymphoid cells. Mucosal Immunol 2014; 7: 842-856.

16. Baird AM, Dockry E, Daly A, Stack E, Doherty DG, O’Byrne KJ, Gray SG. IL-23R is epigenetically regulated and modulated by che motherapy in non-small cell lung cancer. Front Oncol 2013; 3: 162

17. Meng CD, Zhu DD, Jiang XD, Li L, Sha JC, Dong Z, Kong H. Overexpression of interleukin-17 in tumor-associated macrophages is correlated with the differentiation and angiogenesis of laryngea squamous cell carcinoma. Chin Med J (Engl) 2012; 125: 1603-7.

18. Kesselring R, Thiel A, Pries R, Trenkle T, Wollenberg B. Human Th17 cells can be induced through head and neck cancer and have a functional impact on HNSCC development. Br J Cancer 2010; 103: $1245-54$

19. Garley M, Jablonska E, Grabowska SZ, Piotrowski L. IL-17 family cytokines in neutrophils of patients with oral epithelial squamous cell carcinoma. Neoplasma 2009; 56: 96-100.

20. Wu J, Du J, Liu L, et al. Elevated pretherapy serum IL17 in primary hepatocellular carcinoma patients correlate to increased risk of early recurrence after curative hepatectomy. PLoS One 2012; 7: e50035.

21. Huang C, Fu ZX. Localization of IL-17+Foxp3+T cells in esophageal cancer. Immunol Invest 2011; 40: 400-12.

22. Rogala E, Nowicka A, Bednarek W, Barczyński B, Wertel I, Zakrzew ski $M$, Kotarski J. Evaluation of the intracellular expression of interleukin 17 in patients with ovarian cancer. Ginekol Pol 2012; 83: 424-8.

23. Zhang GQ, Han F, Fang XZ, Ma XM. CD4+, IL17 and Foxp3 expression in different pTNM stages of operable non-small cell lung cancer and effects on disease prognosis. Asian Pac J Cancer Prev 2012; 13: 3955-60.

24. Hus I, Bojarska-Junak A, Chocholska S, Tomczak W, Woś J, Dmoszyńska A, Roliński J. Th17/IL-17A, which can play a protective role in the resistance of chronic lymphocytic leukemia. PLoS One 2013; 8: e78091.

25. Yao J, Liu L, Yang M. Interleukin-23 receptor genetic variants contribute to susceptibility of multiple cancers. Gene 2014; 533: 21-5.

26. Zhou S, Ruan Y, Yu H, Chen Y, Yao Y, Ma Y, Gao Y. Functional IL-23R rs10889677 genetic polymorphism and risk of multiple solid tumors: a meta-analysis. PLoS One 2013; 8: e80627.

27. Choi IK, Li Y, Oh E, Kim J, Yun CO. Oncolytic adenovirus expressing IL-23 and p35 elicits IFN- $\boldsymbol{\gamma}$ - and TNF- $\alpha$-co-producing T cell-mediated antitumor immunity. Plus One 2013; 8: e67512.

28. Teng MW, Vesely MD, Duret $\mathrm{H}$, et al. Opposing roles for IL-23 and IL-12 in maintaining occult cancer in an equilibrium state. Cancer Res 2012; 72: 3987-96.

29. Cui G, Yuan A, Goll R, Florholmen J. IL-17A in the tumor microenvironment of the human colorectal adenoma-carcinoma sequence. Scand J Gastroenterol 2012; 47: 1304-12.

30. Omrane I, Baroudi O, Bougatef K, et al. Significant association between IL23R and IL17F polymorphisms and clinical features of colorectal cancer. Immunol Lett 2014; 158: 189-94.

31. Lee HH, Yang SS, Vo MT, et al. Tristetraprolin down-regulates IL-23 expression in colon cancer cells. Mol Cells 2013; 36: 571-6.

32. Santoni M, Massari F, Amantini C, et al. Emerging role of tumor-associated macrophages as therapeutic targets in patients with metastatic renal cell carcinoma. Cancer Immunother 2013; 62: 1757-68.

33. Das Roy L, Pathangey LB, Tinder TL, Schettini JL, Gruber HE, Mukherjee P. Breast-cancer-associated metastasis is significantly increased in a model of autoimmune arthritis. Breast Cancer Res 2009; 11: R56.

34. Starska K, Forma E, Lewy-Trenda I, et al. Expression of CTLA-4 and Foxp3 in peripheral blood T cells of patients with squamous cell laryngeal carcinoma. Contemp Oncol (Pozn) 2013; 17: 370-7.

\section{Address for correspondence}

Katarzyna Starska MD, PhD

Department of Otolaryngology and Laryngological Oncology Medical University of Lodz

Kopcinskiego 22

90-153 Lodz, Poland

tel./fax +48426785785

e-mail: katarzyna.starska@umed.lodz.pl

Submitted: 21.06 .2014

Accepted: $\quad 31.01 .2015$ 